\title{
Osteomyelitis complicating secondarily infected atopic eczema: two case reports and a narrative literature review
}

\author{
Josiah T. Masuka ${ }^{1,2^{*}}$ (D, Katherine Troisi ${ }^{3}$ and Zamambo Mkhize ${ }^{1,2}$
}

\begin{abstract}
Background: Atopic eczema is a relapsing, itchy chronic cutaneous inflammatory disease that commonly affects children. The disease is often complicated by cutaneous infections such as eczema herpeticum, eczema vaccinatum and a varied number of bacterial infections - impetigo, cellulitis and erysipelas. However, rare case reports of infective endocarditis, otitis media and osteo-articular infections have been associated with atopic eczema. These associations possibly represent the extracutaneous infectious complications of atopic eczema.

Case presentation: Here we present two cases of osteomyelitis in HIV negative children with habitual scratching of poorly managed and/or uncontrolled atopic eczema respectively. Both cases presented to the orthopaedic surgeons and were admitted as acute phalangeal osteomyelitis and acute - on - chronic tibial osteomyelitis respectively. The first case was an 8 year old girl who had moderate-severe poorly-controlled atopic eczema and contiguously spread phalangeal osteomyelitis. The second case was an 11 year old pre-pubertal boy who had untreated atopic eczema and tibial osteomyelitis possibly from haematogenously spread Staphylococcus aureus infection. Both were successfully discharged from hospital and currently have well controlled eczema. The 11 year old patient is also being reviewed monthly by the orthopaedic surgeons and is chronic suppressive antibiotics. He may require sequestrectomy, should it be needed.

Conclusions: Invasive staphylococcal and streptococcal osteo-articular (OA) infection can arise as an extracutaneous infectious complication of poorly controlled atopic eczema. It is more common in the 3 to 15 year age group and especially in boys with a septic arthritis to osteomyelitis ratio of around 29:5. Clinicians should maintain a high index of suspicion in patients with moderate-severe atopic eczema and they ought to promptly manage these OA infections with intravenous antibiotics to avoid further complications.
\end{abstract}

Keywords: Osteomyelitis, Osteo-arthritis, Atopic eczema, Secondary infection, Staphylococcus aureus

\section{Background}

Osteomyelitis has rarely been associated with secondarily infected atopic eczema [1-4]. In addition, phalangeal and/or hand osteomyelitis has rarely been described in literature as contiguous contamination secondary infection in atopic eczema or on the hands $[5,6]$. In one reported case series, three cases of acute phalangeal osteomyelitis due to habitual scratching in children with

\footnotetext{
*Correspondence: josiahmasuka@gmail.com

'Department of Dermatology, Nelson R Mandela School of Medicine, Private Bag X7, Congella, Durban 4013, South Africa

${ }^{2}$ Department of Dermatology, Edendale Hospital, 89 Selby Msimang Rd,

Pleissislaer, Pietermaritzburg 3201, South Africa

Full list of author information is available at the end of the article
}

severely infected atopic eczema has been documented [5]. In all three children, no apparent indicators of haematogenous bacterial dissemination such as elevated erythrocyte sedimentation rate (ESR) or pyrexia were observed [7]. On the other hand, invasive staphylococcal bacteremia and haematogenous osteo-articular infections have also been reported sporadically in association with atopic eczema [3, 8, 9]. These cases represent some of the infrequently reported invasive complications of secondarily infected atopic eczema which require prompt and definitive management to prevent further complications [4].

Here we present two unusual cases of osteomyelitis possibly resultant from secondarily infected atopic

(c) The Author(s). 2020 Open Access This article is distributed under the terms of the Creative Commons Attribution 4.0 International License (http://creativecommons.org/licenses/by/4.0/), which permits unrestricted use, distribution, and 
Table 1 Case reports/series reporting osteo-articular infection associated with atopic eczema [modified from [9, 19]]

\begin{tabular}{|c|c|c|c|c|c|c|c|}
\hline Case & Author - Year & Diagnosis & OA type & Age $(Y r)$ & Gender & $\begin{array}{l}\text { Reported atopic } \\
\text { eczema } \\
\text { severity }\end{array}$ & Micro-organism \\
\hline 1 & Patel D; 2015 [4] & $\begin{array}{l}\text { Septic arthritis and osteomyelitis - } \\
\text { left shoulder }\end{array}$ & OAOM & 8 & $\mathrm{~F}$ & Severe & MRSA \\
\hline 2 & Sayaka l; 2013 [21] & Costo-chondral abscess & $\mathrm{OA}$ & 20 & M & NS & NS \\
\hline 3 & Tsutsumi R; 2010 [25] & Cervical spondylitis spondylitis & $\mathrm{OA}$ & 31 & M & Severe & SA \\
\hline 4 & Kitamura S; 2000 [8] & Septic arthritis - hip & OA & 15 & $\mathrm{~F}$ & Recent flare & SA \\
\hline 5 & Boiko S; 1988 [5] & Osteomyelitis - distal phalanx & $\mathrm{OM}$ & 3 & M & Severe & MRSA, Strep \\
\hline 6 & Boiko S; 1988 [5] & Osteomyelitis - distal phalanx & $\mathrm{OM}$ & 4 & $\mathrm{~F}$ & Severe & MSSA, Strep \\
\hline 7 & Boiko S; 1988 [5] & Osteomyelitis - nail plate & $\mathrm{OM}$ & 2 & $\mathrm{~F}$ & Severe & MSSA, Strep \\
\hline 8 & Sharma A; 1997 [2] & Osteomyelitis - fibula & $\mathrm{OM}$ & 4 & M & Severe & MSSA \\
\hline 9 & Nassif A; 1994 [1] & Septic bursitis - mid-tibial, olecranon & $\mathrm{OA}$ & 60 & M & Severe & MRSA \\
\hline 10 & Kusunoki T; 2015 [19] & Right hip osteo-arthritis & $\mathrm{OA}$ & 0.25 & M & Severe & MSSA \\
\hline 11 & Kusunoki T; 2015 [19] & Left hip oste-arthritis & $\mathrm{OA}$ & 11 & M & Recent flare & NS \\
\hline 12 & Numazaki H; 2017 [22] & $\begin{array}{l}\text { *Tibial osteomyelitis } \\
\text { (complication of ACL reconstruction) }\end{array}$ & $\mathrm{OM}$ & 24 & M & Severe & MSSA \\
\hline 13 & Ohno et al.; 2000 [19] & Right sacroiliac & $\mathrm{OA}$ & 13 & M & Moderate-severe & MSSA \\
\hline 14 & Ueda et al.; 2001 [19] & Left hip oste-arthritis & $\mathrm{OA}$ & 3 & M & Unknown & SA \\
\hline 15 & Ono et al.; 2003 [19] & Knee SA & $\mathrm{OA}$ & Infant & Unknown & Moderate-severe & SA \\
\hline 16 & Hidaka et al.; 2004 [19] & Right knee & $\mathrm{OA}$ & 5 & M & Moderate-severe & MRSA \\
\hline 17 & Yamagata et al.; 2004 [19] & Right hip and knee SA & $\mathrm{OA}$ & 12 & M & Moderate-severe & MRSA \\
\hline 18 & Kimura et al., 2005 [19] & Left hip & $\mathrm{OA}$ & 0.25 & $\mathrm{~F}$ & Moderate-severe & MSSA \\
\hline 19 & Nakamura \& Fujioka; 2006 [26] & Left hip & $\mathrm{OA}$ & 5 & M & Moderate-severe & MSSA \\
\hline 20 & Moriwaki et al.; 2006 [19] & Left sacroiliac & $\mathrm{OA}$ & 21 & $\mathrm{~F}$ & Unknown & MSSA \\
\hline 21 & Nakamura \& Fujioka; 2006 [26] & Right knee and femur & $\mathrm{OA}$ & 0.83 & M & Moderate-severe & MRSA \\
\hline 22 & Nakamura \& Fujioka; 2006 [26] & Right hip & $\mathrm{OA}$ & 0.5 & $\mathrm{~F}$ & Moderate-severe & MSSA \\
\hline 23 & Nakamura \& Fujioka; 2006 [26] & Right hip & $\mathrm{OA}$ & 0.58 & M & Moderate-severe & Strep \\
\hline 24 & Hiyane et al.; 2007 [19] & Left hip & $\mathrm{OA}$ & 11 & M & Unknown & NS \\
\hline 25 & Matsushita et al.; 2008 [19] & Left knee & $\mathrm{OA}$ & 1 & $\mathrm{~F}$ & Mild & MSSA \\
\hline 26 & Nagai et al.; 2008 [19] & Left tibia & $\mathrm{OA}$ & 3 & M & Mild & NS \\
\hline 27 & Nagai et al.; 2008 [19] & Left femur & $\mathrm{OA}$ & 3 & M & Moderate-severe & MSSA \\
\hline 28 & Nagai et al.; 2008 [19] & Left hip & $\mathrm{OA}$ & 0.92 & $\mathrm{~F}$ & Moderate-severe & MSSA \\
\hline 29 & Suzuki et al.; 2009 [19] & Right knee & $\mathrm{OA}$ & 7 & $\mathrm{~F}$ & Moderate-severe & SA \\
\hline 30 & Kinugasa et al.; 2009 [19] & Right hip & $\mathrm{OA}$ & 23 & $\mathrm{~F}$ & Unknown & Strep \\
\hline 31 & Hashi et al.; 2012 [27] & Right hip & $\mathrm{OA}$ & 5 & $\mathrm{~F}$ & Moderate-severe & MRSA \\
\hline 32 & Yamagata et al.; 2012 [19] & Right hip & $\mathrm{OA}$ & 12 & M & Moderate-severe & MRSA \\
\hline 33 & Yasuda \& Nisimatsu; 2012 [28] & Right sacroiliac & OA & 15 & M & Moderate-severe & Strep \\
\hline 34 & Kyo; 2014 [23] & Knee & $\mathrm{OA}$ & 13 & M & NS & SA \\
\hline 35 & Kyo; 2014 [23] & Knee & $\mathrm{OA}$ & 27 & M & NS & SA \\
\hline 36 & Current case 1 & Phalangeal osteomyelitis & $\mathrm{OM}$ & 8 & $\mathrm{~F}$ & Mild & NS \\
\hline 37 & Current case 2 & Tibial osteomyelitis & $\mathrm{OM}$ & 11 & M & Severe & NS \\
\hline
\end{tabular}

Abbreviations: OA Osteoarthritis, OM Osteomyelitis, MRSA Methicillin Resistant Staph. Aureus; SA Staph.Aureus, Strep Streptococcus, NS Not specified

eczema in African children. These children were referred on the same day to our dermatology clinic from orthopaedic surgeons for the management of the underlying atopic eczema after initial admission for phalangeal and tibial osteomyelitis respectively. In addition, a narrative literature review of similar case presentations has also been carried out to describe the demographic and clinical features of osteo-articular infections associated with 
atopic eczema. The occurrence of the two presented cases in light of similar previous case reports may not be coincidental and calls upon clinicians to be aware of the potential complications of atopic eczema [4].

\section{Case presentation}

\section{Case 1}

An 8 year old, black female child was referred to the dermatology team from orthopaedic surgeons with a 3 month history of a swollen right index finger. On further enquiry, the patient was noted to be atopic with comorbid chronic asthma and atopic eczema. The child was being managed on aqueous cream baths, topical betamethasone cream, a non-sedating antihistamine - loratadine and liquid paraffin as an emulsifying ointment and an asthma medication pump. On the current dermatology consultation, the child's caregiver mentioned that the child had been scratching the itchy right index finger. In the period prior to the presentation, the child's finger got swollen and was painful prompting the hospital visit and subsequent admission. No history of phalangeal trauma or diabetes mellitus was elicited from the patient's caregiver, which was also confirmed in subsequent tests. The child was HIV negative.

Examination revealed a swollen, mildly fluctuant index and middle phalanges with draining sinuses. There was an eczematous plaque with scaling and no lichenification. The fingers were tender and warm to palpation. No dysmorphic features were observed and the child's body temperature was unremarkable at $37.2{ }^{\circ} \mathrm{C}$. The X-ray findings were consistent with osteomyelitis and the child was admitted by the orthopaedic surgeons for intravenous antibiotics (amoxicillin/clavulanic acid, due to the unavailability of cloxacillin in our institution, which would be the drug of choice) to control the acute infection as surgical drainage was not warranted. The patient was discharged a week later to complete a 1 month oral antibiotic therapy course at home and to continue with her eczema medication. On review, the cellulitis had healed and the eczema lesions had been unmasked. The phalanges displayed the eczematous plaque with diffusely demarcated borders. No weeping or crusting was observed. Her initial calculated (Eczema Area and Severity Index) EASI score and severity levels on presentation were 2.00 and mild severity respectively using the classification proposed by Leshem et al. [10]. The patient is now being followed up in the dermatology out-patient clinic for her atopic eczema.

\section{Case 2}

An 11 year old, black male child was seen as a referral to the dermatology clinic at Edendale hospital, Pietermaritzburg with an 8 month history of bilateral leg swelling. The lesions started as 'small pimples' which subsequently drained pus and the legs got swollen and painful. Further enquiry showed that the patient had developed an itchy rash on both legs 10 months prior to the current presentation. No medical care had been sought, but the patient was applying a topical, traditional medicine to the legs with no relief of the symptoms. A history of trauma to the right leg about 5 months prior to the current presentation was noted in the patient's history, but the other details were sketchily recalled by the patient. The rest of his past medical history was unremarkable with no previous history of atopy or diabetes mellitus.

Confluent, weeping plaques with diffuse edges and post-inflammatory hyperpigmentation were observed together with excoriations and a chronic draining sinus on the right leg. The left leg was swollen and erythematous, but had no sinus. The legs were tender on palpation, however there was full range of motion on both the ankle and knee joints bilaterally with normal pedal pulses. On the day of admission, the patient was apyrexial and he remained apyrexial during the period under admission. The complete blood count showed an unremarkable leucocyte count, $8.65(4-10)$ cells $/ \mathrm{mm}^{3}$ and a slight decrease in the haemoglobin count of $11.8 \mathrm{~g} / \mathrm{dl}$ $(12-15 \mathrm{~g} / \mathrm{dl})$. There was a marked increase in the markers of inflammation with an Erythrocyte Sedimentation Rate (ESR) of $45(0-10) \mathrm{mm} /$ hour, C Reactive Protein (CRP) $30(0-5) \mathrm{mg} / \mathrm{L}$ and a platelet count of 500 (150-400) cells $/ \mathrm{mm}^{3}$. The X-ray findings were consistent with chronic osteomyelitis of the right tibia, the left tibia X-rays were normal. The patient was HIV negative. However no pus swab was recorded in the patient's charts. His initial calculated EASI score and severity levels on presentation were 9.60 and moderate severity respectively [10].

The patient was initially treated by orthopaedic surgeons as an acute-on-chronic osteomyelitis and intravenous amoxicillin/clavulanic acid was administered to manage the acute sepsis together with paracetamol as analgesia. On review by the dermatology team, the diagnosis was modified to chronic osteomyelitis secondary to infection with underlying chronic eczema. Potassium permanganate baths, topical betamethasone, silversulphur diazine, an emulsifying ointment and a nonsedating antihistamine - loratadine were added to his medical management. The patient was subsequently discharged to complete a course of rifampicin and trimethoprim/sulfamethoxazole, (orthopaedics current chronic osteomyelitis protocol for older children/adult patients) and was to be reviewed in both the dermatology and orthopaedic out-patient clinics. Further orthopaedic management has consisted of monthly clinic reviews, chronic suppressive antibiotics and sequestrectomy might be considered, should it be needed. 


\section{Discussion and conclusions}

In this case series, we have presented two unusual cases of osteomyelitis associated with severely infected atopic eczema in HIV uninfected children. The cases represent two of the three generally accepted mechanisms of osteomyelitis infection - contiguous spread and haematogenous spread [11]. The first case developed possibly after direct infection of the distal phalangeal bone due to an overlying septic eczema focus. The second case possibly had haematogenous spread to the tibial metaphysis. This was secondary to a bacteraemia resultant from the septic eczema, which then settled in the metaphysis as commonly happens in immature long bones [12, 13]. Both children had habitual, excessive scratching of their dry, fissured skin in poorly or untreated eczema respectively. In addition, there were no other apparent sources of infection in both cases.

The phalangeal osteomyelitis case is similar to three cases reported by Boiko et al. [5]. There was insidious onset of radiologically confirmed osteomyelitis without any associated fever or ESR elevation. This signified a localized infection as opposed to the second case which presented with tibial osteomyelitis associated with a raised ESR. The raised ESR in the second case indicates haematogenous spread, possibly of invasive staphylococcal infection presenting as osteomyelitis [5]. Even though no bacterial cultures were done prior to initiation of antibiotic therapy in both cases, we strongly suspect that Staphylococcus aureus was the causative organism. The lack of bacterial culture results may be a major limitation to our study. However, staphylococcal bacterial colonization has been shown to be more common in atopic eczematous skin compared to normal skin [14].

Furthermore, it has been postulated that recurrent bacterial and viral infections often complicate atopic eczema possibly due to an interplay between staphylococcal enterotoxins (super-antigens), cutaneous barrier defects and the dysfunctional cutaneous innate immune system [15]. The latter is characterized by increased skin $\mathrm{pH}$ and decreased antimicrobial peptides - human $\beta$ defensins and cathelicidins coupled to increased $\mathrm{CD}_{4}^{+}$ Th2 cytokines - IL-4 and IL-13 [15, 16]. Decreases in antimicrobial proteins are caused by the skewing of the lymphoctic response towards the $\mathrm{CD}_{4}^{+} \mathrm{Th} 2$ direction with increased cytokines IL-4 and IL-13 resultant from increased keratinocyte derived thymic stromal lymphoprotein expression in atopic eczema [15]. Additionally, the relapsing, itchy chronic inflammation of atopic eczema is also perpetuated by the reduced staphylococcal bacterial inhibition and low "natural moisturizing factor" (NMF) resultant from the lack-of-function filaggrin gene defects $[15,17]$. The reduced NMF exacerbates atopic eczema due to the dry skin aggravating and perpetuating the "itch - scratch" cycle $[15,18]$. Chronic scratching of the itchy skin potentially worsens the already weak cutaneous barrier, thereby facilitating the entry of allergens and pathogens into the skin $[4,19]$. Consequently, cutaneous and extra-cutaneous infections occur with higher incidence in atopic eczema patients compared to nonatopic eczema patients [20]. Eczema herperticum, erysipelas, impetigo, cellulitis, otitis media and streptococcal throat infection have been widely documented in patients with atopic eczema [15, 17, 20].

However, clinicians need to keep a high index of suspicion for invasive staphylococcal infection especially in toxaemic eczematous patients $[8,16]$. Systemic infections such as osteo-articular infections, infective endocarditis and pneumonia have rarely been diagnosed in association with eczema $[1,2,5,9,16]$. Overall, a total of at least 35 case reports of invasive osteo-articular infection have been published in association with atopic eczema including 6 case reports first collated in 2005 by Benenson et al. [9]. Most of these retrieved case reports, $30(85.71 \%)$ have been reported in Japanese patients with the remainder described in other settings. Twenty nine (29) out of the 35 case reports were on septic arthritis whilst 5 were on osteomyelitis and 1 case report had both osteomyelitis and septic arthritis [4, 9, 19, 21-23]. Septic-arthritis mostly involved the hip joint 14 (48.28\%) followed by the knee joint 8 (27.59\%) Table 1.

The majority of the osteo-articular cases, 28 (80.0\%) were observed in paediatric patients below 18 years of age whilst the remainder was mostly in young adults. The median and average ages of the patients described in the case reports were 6 (IQR; $2.75: 15.0$ ) years and $10.72 \pm 12.20$ years respectively with a $22: 12 \mathrm{M}$; F ratio. The mode of the presumed severity level of the atopic eczema on presentation with osteo-articular disease was moderate - severe disease. Staphylococcus aureus was cultured in $28(80.0 \%)$ whilst streptococcus ( $\beta$ haemolytic group B streptococcus or Streptococcus viridans) was cultured in $6(17.14 \%)$ of the retrieved case reports. Methicillin resistant and methicillin sensitive Staphylococcus aureus were documented in 8 (22.86\%) and 13 (37.14\%) case reports of osteo-articular infection respectively. Both Staphylococcus aureus (all MRSA) and streptococcus were both cultured in $3(8.57 \%)$ whilst the culture result was unknown in $4(11.43 \%)$ of the case reports.

Japanese individuals predominated in the retrieved case reports [19] possibly because of the increased use of sensitive Magnetic Resonance Imaging (MRI) and bone scans [23, 24] for patient work-up in Japan compared to Africa for instance. Nevertheless, a significant number were also observed in other populations and were associated with osteomyelitis. This underscores the need for aggressive management of the underlying atopic eczema and a high index of suspicion for osteo-articular infections as they can cause growth disturbances, deformities 
and even death [24]. This is especially so in patients who present with a persistent fever, an elevated ESR and/or moderate-severe or flaring eczema as there might be secondary staphylococcal or streptococcal infection of the eczematous lesions sometimes with subsequent systemic spread $[4,9,17]$.. Early and appropriate management of the systemic infections with intravenous antibiotics [23] may reduce the indirect health costs associated with the potential atopic eczema associated systemic infection.

\section{Abbreviations \\ CD: Cluster of Differentiation; CRP: C Reactive Protein; ESR: Erythrocyte Sedimentation Rate; HIV: Human Immunodeficiency Virus; NMF: Natural moisturizing factor}

\section{Acknowledgements}

The authors are grateful for the assistance provided by Sr Z Ndlovu and Sr L Ndaba and other staff at Edendale Hospital in preparing this manuscript.

\section{Authors' contributions}

$J M, K T$ and ZM collected the clinical information about the two cases. JM drafted the manuscript with input/modifications from KT and ZM. All the authors reviewed the manuscript and approved the final version.

\section{Funding}

No external sources of funding were used for this study.

\section{Availability of data and materials}

The datasets used and/or analysed during the current study are available from the corresponding author on reasonable request.

\section{Ethics approval and consent to participate}

This case report was approved by the Edendale Hospital Ethics Committee. All patient data was de-identified.

\section{Consent for publication}

Written informed consent for publication of the patients' clinical details and/ or clinical images was obtained from the patients/guardians/ relative of the patients. Copies of the consent forms are available for review by the Editor of this journal.

\section{Competing interests}

The authors declare that they have no competing interests.

\section{Author details}

'Department of Dermatology, Nelson R Mandela School of Medicine, Private Bag X7, Congella, Durban 4013, South Africa. ${ }^{2}$ Department of Dermatology, Edendale Hospital, 89 Selby Msimang Rd, Pleissislaer, Pietermaritzburg 3201, South Africa. ${ }^{3}$ Department of Orthopaedics, Edendale Hospital, 89 Selby Msimang Rd, Pleissislaer, Pietermaritzburg 3201, South Africa.

Received: 11 September 2019 Accepted: 22 December 2019 Published online: 03 February 2020

\section{References}

1. Nassif A, Smith D, Hanifin J. Olecranon and pretibial bursitis: coincidence or association. J Am Acad. 1994:30:737-42.

2. Sharma A. Atopic dermatitis and stapylococcus aureus - induced osteomyelitis - a peculiar association in a case. Pediatr Dermatol. 1997;14(6): 453-5.

3. Carr T, Avila P. Recurrent deep-seated staphylococcus aureus infections of unclear aetiology. J Clin Immunol. 2011;31:S29.

4. Patel D, Jahnke M. Serious complicatications from staphylococcal aureus in atopic dermatitis. Pediatr Dermatol. 2015;32(6):792-6.

5. Boiko S, Kaufman R, Anne W, Lucky A. Osteomyelitis of the distal phalanges in three children with severe atopic dermatitis. Arch Dermatol. 1988:124: 418-23.

6. Pinder R, Barlow G. Osteomyelitis of the hand. J Hand Surg Eur Vol. 2016; 41(4):431-40. https://doi.org/10.1177/1753193415612373.
7. Nade S. Acute haematogenous osteomyelitis in infancy and childhood. J Bone Joint Surg Br. 1983:65:109-19.

8. Kitamura S, Nakayam Y, Shirai Y, Hashiguchi S, Kim R. Septic atrhritis of the hip associated with atopic dermatitis - a case report. J Nippon Med Sch. 2000;67(6):464-7.

9. Benenson S, Zimhony O, Dahan D, Solomon M, Raveh D, Schesinger Y, Yinnon A. Atopic dermatitis - a risk factor for invasive staphylococcal aureus infections:two cases and review. Am J Medicine. 2005;1 18(9):1048.

10. Leshem Y, Hajar T, Hanifin J, Simpson E. What the EASI score tells us about the severity of atopic dermatititis - an interpretability study. Br J Dermatol. 2015:172(5):1353-7.

11. Birt M, Anderson D, Toby E, Wang J. Osteomyelitis: recent advances in pathophysiology and therapeutic strategies. J Ortho. 2017;14:45-52

12. Paakkonen M, Kallio M, Peltola H, Kallio P. Antibiotic treatment and surgery for acute haematogenous calcaneal osteomyelitis of childhood. J Foot Ankle Surg. 2015;54:840-3.

13. Francis J, Robson J, Wong D, Walsh M. Chronic recurrent multifocal Q fever osteomyelitis in children: an emerging clinical challenge. Pediatr Infect Dis J. 2016:35(9):972-6.

14. Higaki S, Morohashi M, Yamagashi T, Hasegawa Y. Comparative study of staphylococci from the skin of atopic dermatitis patients and from healthy subjects. Int J Dermatol. 1999:38(4):265-9.

15. Ong $P$, Leung $D$. Bacterial and viral infections in atopic dermatitis: a comprehensive review. Clinic Rev Allerg Immunol. 2016;51(3):329-37.

16. Tsuboi I, Yumoto T, Toyokawa T, et al. Staphylococcus aureus bacteremia complicated by ppsoas abscess and infective endocarditis in a patient with atopic dermatitis. Case Rep Infect Dis. 2017;2017:1-4. https://doi.org/10. 1155/2017/4920182

17. James W, Elston D, Berger T, Neuhaus I. Andrews' diseases of the skin: clinical dermatology. 12th ed. Philadelphia: Elsevier; 2016.

18. Murota $\mathrm{H}$, Katayama I. Exacerbating factors of itch in atopic dermatitis. Allergol Int. 2016;66(1):8-13.

19. Kusunoki T, Shimozono F, Maruki M, Futami T, Fujii T. Septic arthritis and atopic dermatitis: 2 cases and a review of the recent literature. J Investig Allergol Clin Immunol. 2015;25(3):214-36.

20. Narla S, Silverberg J. Association between childhood atopic dermatitis and cutaneous, extracutaneous and systemic infections. Br J Dermatol. 2018:178: $1466-9$

21. Sayaka I, Kenichi K, Hideaki T, Miwa M, Tomoya F, Katsuya E. A case of severe atopic dermatitis complicated by costal costal cartilage junction abscess. J Japan Prim Care Assoc. 2013;36(4):315-7.

22. Numazaki H, Kobbayashi H, Yoshida K, Hakozaki M, Konno S. Prolonged infection at the tibial bone tunnel after anterior cruciate ligament reconstruction. Fukushima J Med Sci. 2017:63(2):121-5.

23. Kyo H, Hayashi M, Yamawaki Y, Watanabe M, Okuntani Y, Kanamura M, Takeuchi H, Ota S, Onishi E, Iwaki K, et al. Atopic dermatitis as a potential portal of septic arthritis. J Arthritis. 2014;3:135.

24. Yeo ARM. Acute haematogenous osteomyelitis in children. BMJ. 2014:348: g66.

25. Tsutsumi REA. A case of cervical spondylitis spondylitis with Staphylococcus aureus bacteremia that is considered to be the infection route of severe atopic dermatitis skin lesions. Neurology. 2010;5:516-8.

26. Nakamura K, Fujioka F. Septic arthritis in children. Jap J Pediatr. 2006;59:11520.

27. Hashi R, Miyake F, Takehara A, Sukamoto A, Yamauchi J, Wada K, Nakauchi S, Ohshima T. Five cases of septic arthritis in childhood. Jap J Pediatr. 2012; 65:2217-21.

28. Yasuda T, Nisimatsu H. Acute sacroiliac joint infection in a rugby player with atopic dermatitis. Clin J Sport Med. 2012;22:508-10.

\section{Publisher's Note}

Springer Nature remains neutral with regard to jurisdictional claims in published maps and institutional affiliations. 